\title{
LTE MODELS
}

\author{
ROBERT L. KURUCZ \\ Harvard-Smithsonian Center for Astrophysics \\ 60 Garden St., Cambridge, MA 02138, USA
}

\begin{abstract}
I can compute arbitrary-abundance models and spectra at high resolution using millions of atomic and diatomic molecular lines. Examples are given for Sakurai's object and for a $\lambda$ Boo star. I am continuing to improve the input line data.
\end{abstract}

\section{Introduction}

ATLAS12 is an opacity sampling program that I originally wrote in 1992 to allow computation of models with individual abundances. The program reads data for 58 million atomic and diatomic molecular lines from my CD-ROMs 1 and 15. I have computed sample models for Sirius (Kurucz 1993), Vega (Castelli and Kurucz 1993), Sakurai's object, $\lambda$ Boo stars, and various B stars. ATLAS12 can be used to model exciting stars for H II regions with abundances consistent with those of the $\mathrm{H}$ II region. I have recently fixed problems with convection and have computed models for the Sun, Arcturus, and Betelgeuse. Those models are not final but currently are grids of models being used to search for the best fit. As I do not yet have line data for tritomic molecules, I cannot yet compute. cooler models. Here I will show only examples for Sakurai's object and for a $\lambda$ Boo star.

The fluxes predicted by ATLAS12 are not accurate in intermediate or narrow bandpass intervals because the sample size is too small. A stripped version of the spectrum synthesis program SYNTHE (Kurucz and Avrett 1980) is used to generate resolution 500000 flux for the converged model using the same line data that were used in the model calculation. The interval can be as large as $10 \mathrm{~nm}$ to $300 \mu \mathrm{m}$ or just a small region of interest. As most of the line positions are predicted, the computed flux spectrum is not realistic when compared to a high resolution observed spectrum. For that case the spectrum can be computed using the full SYNTHE with only the subset of lines with accurate wavelengths. The spectrum calculations are not coupled to ATLAS12; any model can be used.

The high resolution spectrum can be used directly or can be instrumentally broadened io compare to low resolution observations. It is now feasible to compute grids of "observables" by computing sections of spectra for every model in a grid. A version of SYNTHE is being developed for pretabulating distribution function opacity so that grids of models can be produced quickly with ATLAS9 (CD-ROM 13).

In 1995 I published CD-ROM 23, Atomic Line Data (Kurucz and Bell) with all the laboratory and computed line data with good wavelengths sorted into one file, and then also divided into 10 $\mathrm{nm}$ or $100 \mathrm{~nm}$ blocks for convenience. I have made no significant progress on the line data in the last two years because of problems with office space and computers. I now have an Alpha and several Vaxes with $60 \mathrm{~GB}$ of disk, a CD writer, and a web server. There are directories for every atom and diatomic molecule. I will soon begin large scale production of atomic and molecular line data. I will continue to distribute the results on CD-ROMs, and I will make them available on the World Wide Web.

\section{Sakurai's Object}

To demonstrate that ATLAS12 really is working I will outline the steps in the construction of a model for the very chemically peculiar star Sakurai's object. There are two posters on it at this General Assembly (Kerber, Gratl, Kimeswenger, and Weinberger 1997; and Gautschy, Duerbeck, 
van Genderen, and Benetti 1997). It is a late He-flash star that is rapidly evolving. S. Benetti asked me to try to compute models for the time when it had approximately Teff $=7500 \mathrm{~K}$, log $\mathrm{g}=0.5, \mathrm{Vturb}=9 \mathrm{~km} / \mathrm{s}$, and abundances $\mathrm{H}=.02, \mathrm{He}=.94, \mathrm{C}=.02, \mathrm{O}=.01, \mathrm{Mg}=.00002, \mathrm{Al}=.00002$, $\mathrm{Si}=00005$, and $\mathrm{Fe}=.00001$. In order to model a star one normally must construct a grid of models varying each parameter to determine the sensitivity of the model and spectrum to each parameter, and then choose the best fit. However in this case I computed only four models as a test, starting with $7500,1.0$, then $7250,0.5,7500,0.5$, and $7750,0.5$. ATLAS12 computes opacity at 30000 points to determine flux integrals. Since it has no pre-existing knowledge of Rosseland opacity, it cannot scale models. It must have a close starting guess or it will not converge. I try various models from preexisting grids until I find something that works. In this case I finally used the $7500,1,[+1.0], 2 \mathrm{~km} / \mathrm{s}$ model from CD-ROM 13, a 10 times solar abundance model. ATLAS12 requires an individual abundance for each element. First ATLAS12 edited the line list. It eliminated all lines that cannot possibly be significant at any layer of the atmosphere. It made a file of the remaining few million lines. That file is used for all the variational models and in subsequent spectrum calculations with SYNTHE. The sampled fluxes are computed and added to the total flux integrand and discarded. The program iterates to convergence. The emergent flux is determined by spectrum synthesis at resolution 500000 .

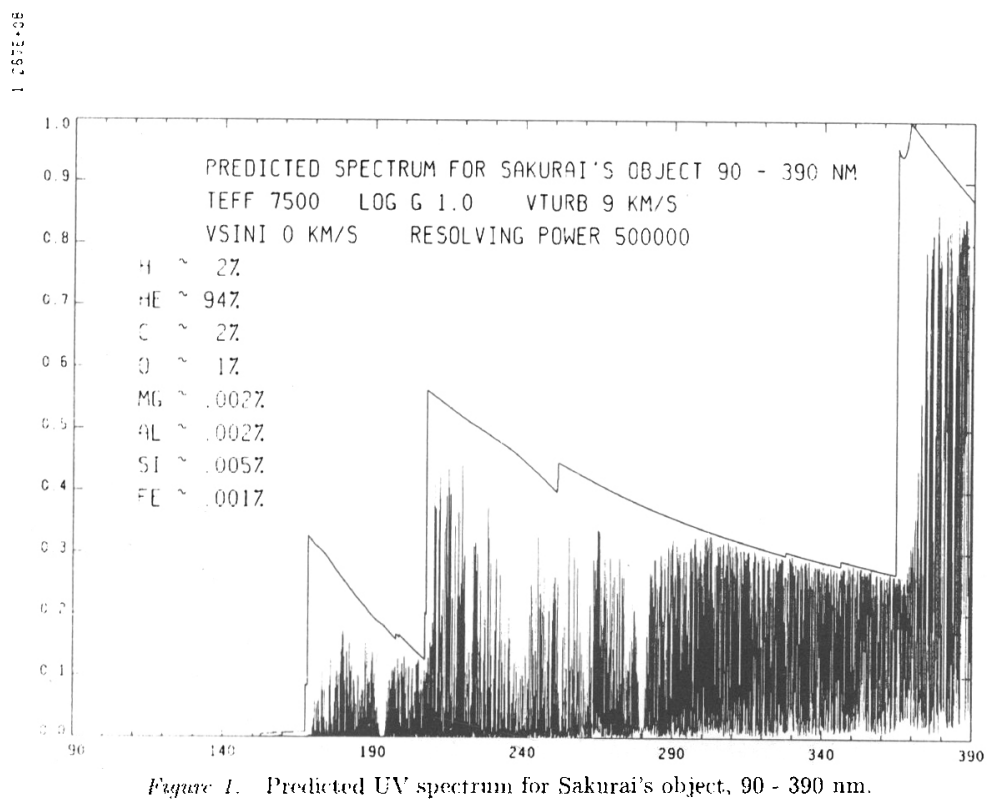

Figures 1 and 2 show the spectrum computed for the first model. The high resolution spectrum can then be degraded to any observational resolution. Since most of the lines have predicted wavelengths, comparison to a high resolution observed spectrum requires recomputing the spectrum using only the lines with good wavelengths. The first model was used as the starting model for subsequent models. However, if the change in parameters is too large, the new models do not converge. It may be necessary to compute an intermediate model, say at $7250 \mathrm{~K}$, before going down to $7000 \mathrm{~K}$. Since ATLAS12 seems to work (i.e. produces a publishable result), it may work for your favorite star as well. (Actually $\mathrm{O}^{-}$and $\mathrm{C}^{-}$may be important for Sakurai's object. I am adding them to the programs.)

\section{Sample $\lambda$ Boo Star}

I am computing grids of ATLAS12 models with $\lambda$ Boo-like abundances for Michele Gerbaldi. Nicole Allard (Allard, Kurucz, Gerbaldi, and Faraggiana 1997) has computed new Lyman $\alpha$ wing opacities 


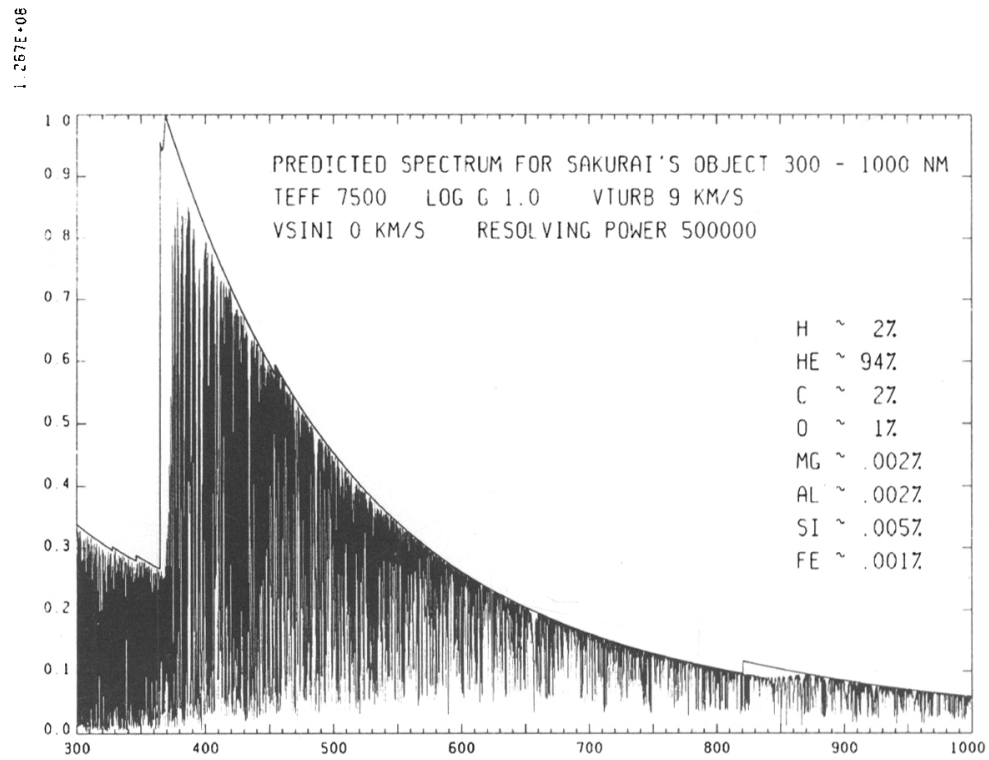

Figure 2. Predicted visible spectrum for Sakurai's object, $300-1000 \mathrm{~nm}$. Note the strong Paschen series merging into the Paschen continuum even with a low $\mathrm{H}$ abundance.

with improved quasi- $\mathrm{H}_{2}$ and quasi- $\mathrm{H}_{2}^{+}$resonances that have been added to ATLAS12 and SYNTHE. Figures 3 show the striking effect on the ultraviolet spectrum.

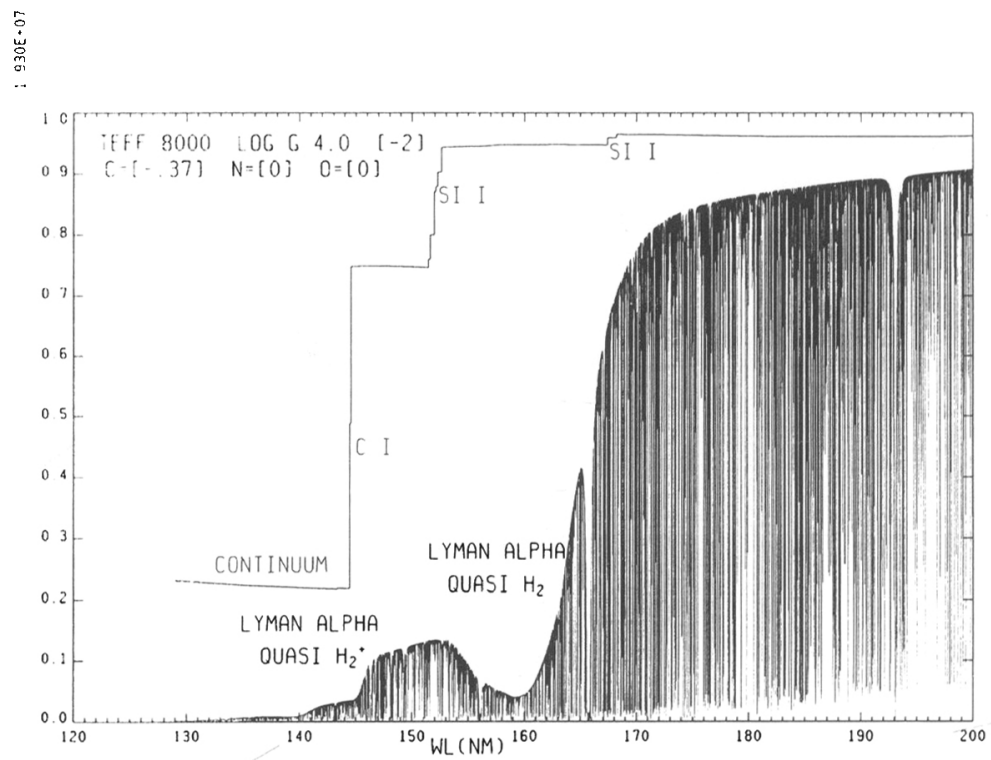

Figure 3. UV spectrum for a model with the $\lambda B o 0$ abundance pattern showing the strong Lyman $\alpha$ resonance features. 


\section{References}

Allard, N.F., Kurucz, R.L., Gerbaldi, M., and Faraggiana, R. (1997) in preparation.

Castelli, F. and Kurucz, R.L. (1993) in Peculiar versus Normal Phenomena in A-type and Related Stars (eds. M.M. Dworetsky, F. Castelli, and R. Faraggiana), A.S.P. Conference Series 44, pp. 496-501.

Gautschy, A., Duerbeck, H.W., van Genderen, A.M., and Benetti, S. (1997) General Assembly abstract book p. 41. Kerber,F., Gratl, H., Kimeswenger,S., and Weinberger, R. (1997) General Assembly abstract book p. 41.

Kurucz, R.L. (1993) in Peculiar versus Normal Phenomena in A-type and Related Stars (eds. M.M. Dworetsky, F. Castelli, and R. Faraggiana), A.S.P. Conference Series 44, pp. 87-97.

Kurucz, R.L. and Avrett, E.H. (1981) SAO Special Report No. 391. 139 pp. 